\title{
Ventilación oscilatoria de alta frecuencia en niños con síndrome de dificultad respiratoria "del adulto"
}

\author{
José I. Rodríguez C. ${ }^{\text {; }}$ Ricardo Ronco M. ${ }^{1}$; Andrés Castillo M. '; Gustavo Guzmán R. ${ }^{\text {; }}$ \\ Astrid Valenzuela S.'; Cecilia Perret P. '; Valeria Acevedo A. '; Enrique Paris M. ${ }^{1}$
}

\begin{abstract}
Resumen
Objetivos: evaluar la utididad de la ventiación de alta frecuencia oscilatoria en el sindrome de dificuliad res. piratoria cgucia pediárico. Pacientes y méfodo: once niños de 3 días a 8 oños (media 22.1 meses) con doño putmonar difuso, en Iralamiento con ventíación mecánica convencional e indice de oxígenoción mayor a 20 (42 $\mathbf{t}$ 12,2) fueron somelidos en tarma prospectiva no controlocia a ventilación de alia frecuencia oscitotoria con volumen pulmonar elevado. Resultodos: al índice de oxigenación disminuyó significativamente a $22 \pm 9.6$ en las primeras $4 \mathrm{~h}$ con ventilación de clta frecuencio. Posleriormenle la disminución fue sostenida aunque no significativa. Ocho pacienles sobrevivieron y fueron extubados desde ventilación mecánica convencionat. De los tres follecidos. dos mostraron buena respuesto a la ventilación de olta frecuencia, y ningune de las muerles se relocionó disectamente con la fallo respiratorio. La letolidad de estos pacientes, estimada originalmente en más de dos tercios, se redujo a menos de un tercio, come en otras series pediálricas de vertilación con alta frecuencia oscilatoria. No se registroron complicaciones alribuibles al procedimierto. Conchusiones. la ventilación oscilatoria de alla frecuencia porece una opción eficaz y sagura en niños con daño pulmonar agudo de diferentes causas.
\end{abstract}

(Palabras clave: síndrome de dificuliad respiratoria adulio, respiración artificıol, ventilación mecónıca, alıa fre. cuencia, oscílatorio.t

\section{High frequency oscilatory ventilation in pediatric patients with "adult" type respiratory distress syndrome}

Objectives: 10 asses usefulness of high frequency oscillatory ventilation in children with "adult rype" acute respiratory distress syndrome. Patients and methods: eleven infants and children aged 3 days 108 years imean 22.1 monthl with difuse lung iriury and oxygenalion index over $20142 \pm 12.21$, were non candomly ventilaled with high irequency oscilatory ventilation and o high pulmonory volume. Results a significant decreose in oxigenarion index to $22 \pm 9.6$ was recorded in the first four hours under high kequency ventilation and this reduction was sustained. Eight oul of 11 patients survived, all of them were able 10 be relurned to conventional mechanical ventilolion and were eventually extubated. Three patients died, no deaths were due to respiratory failure. Predicted montily among these patients wos $70 \%$, however $72.7 \%$ survival wos recorded. There were no side effects atributable to high frequency oscilatory ventitation. Conclusion: in pediatric patients with severe aduli type respiratory distress syndrome of different causes, high frequency oscilatory ventilolion may be o sate and effeclive lool.

(Key words: respiratory distress syndrome, adjlt, high frequency ventilation, respiration, artificial.)

La ventilación de alta frecuencia consiste en la aplicación de procedimientos de apoyo a la

1. Unidad de Cuidados Intensivos, Servicio de Pediatría, Hospital Clínico Pontificia Univerșidad Católica de Chile. respiración que emplean frecuencias respiratorias mayores que las normales (más de $60 \mathrm{cpm}$ ) y volúmenes corrientes cercanos al espacio muerto ${ }^{1,2}$. Su introducción en clínica es reciente. Su propósito es disminuir el daño pulmonar inducido por el volumen en la asistencia ventila- 
toria mecánica (volutrauma) ${ }^{3.7}$ en pacientes con insuficiencia respiratoria aguda (IRA) grave ${ }^{5,7}$.

Las experiencias iniciales con ventilación de alta frecuencia se desarrollaron en recién na$\operatorname{cidos}^{8 \cdot 10}$, donde hoy su uso es corriente, especialmente en la modalidad oscilatoria ${ }^{10.11}$, que se distingue de las otras variedades de ventilación a alta frecuencia por proporcionar una fase espiratoria activa $2,7,11$.

Los primeros informes exitosos de su empleo en lactantes y niños mayores datan de $1993^{12.14}$. Si bien las experiencias publicadas son aûn escasas $^{12,14.17}$, y en pacientes de estas edades con insuficiencia respiratoria grave la sobrevida con el procedimiento no parece mayor que con la asistencia ventilatoria mecánica convencional, la ocurrencia de daño pulmonar es menor ${ }^{15}$.

El objetivo de esta comunicación es describir el empleo, la eficacia y seguridad de la ventilación oscilatoria de alta frecuencia (VAFO) en niños con IRA grave, más allá del período neonatal.

\section{Pacientes y método}

En forma prospectiva no controlada se aplicó el procedimiento mediante un equipo Sensormedics( $3100 \mathrm{~A}$ en I I pacientes ( 6 varones) con insuficiencia respiratoria aguda, daño pulınonar difuso agudo que requerfa ventilación mecánica (VM) o síndrome de fuga aérea, índice de oxige. nación (IO = PMVA $\times \mathrm{FiO}_{2} \times 100 / \mathrm{PaO}_{2}$, donde $\mathrm{PMVA}=$ presión media de la via adrea) $>20$ y relación $\mathrm{PaO}_{2} \mathrm{FiO}_{2}<$ 200. Se excluyeron niños con enfermedad bronquial obstructiva aguda, hipotensión arterial refractaria. flujo pulmonar pasivo.

Antes de aplicar la ventilación de alta frecuencia se registraron -en todos los pacientes- los reglajes ventilatorios empleados en el método convencional; las concentraciones de gases arteriales, el IO, la $\mathrm{PaO}_{2} / \mathrm{FiO}_{2}$, la diferencia alvéolo arterial de oxígeno $\left(\mathrm{AO}_{2}-\mathrm{aO}_{2}\right)$ y se estimaron el índice de dà̃o pulmonar agudo -a partir de la radiogafía de tórax ${ }^{18}$ - y el júmero de sistemas comprometidos ${ }^{14}$.

La ventilación mecánica convencional se realizó -en todos los casos- con modos controlados por presión mediante ventilador Siemens $900 \mathrm{C}$ hasta obtener $\mathrm{PaCO}_{2} \leq 65$ con $\mathrm{pH} \geq 7,25$ y saturaciones de oxígeno (Sat $\mathrm{O}_{2}$ ) $\geq 90 \%$, para lo cual la presión media de la vía aérea (PMVA) se aumentó limitando la presión inspiratoria máxima (Pim) hasta $535 \mathrm{~cm} \mathrm{H} \mathrm{H}_{2} \mathrm{O}$. Inicialnente se aumentó progresivamente la presión positiva al final de la espiración (Pecp) hasta $8 \mathrm{~cm} \mathrm{H}_{2} \mathrm{O}$ y, si esto no fue suficiente, se invirtió la relación inspiración: espiración (I: E), hasta un máximo Peep + autopeep de $15 \mathrm{~cm} \mathrm{H}_{2} \mathrm{O}$. En los casos en que se in. virtió la relación $\mathrm{l} / \mathrm{E}$ se usó sedación y parálisis.

La ventilación oscilatoria de alsa frecuencia se realizo con los pacientes sedados y paralizados con benzodiazepinas y vecuronio en infusión continua $y$ con volumed pul- monar elevado $11,12.15$, iniciando el ciclo de alta frecuencia con presiones medias de via aérea 6 a $8 \mathrm{~cm} \mathrm{H}_{2} \mathrm{O}$ más altas $y$ amplitudes de la presión inpiratoria máxima $(\Delta \mathrm{P}) 10 \mathrm{~cm}$ $\mathrm{H}_{2} \mathrm{O}$ sobre tas de la ventilación mecánica convencional, constatando movimiento ascendente del tórax. La frecuencia respiratoria (FR) se ajustó al peso del paciente (2 a 5 $\mathrm{kg}: 10 \mathrm{~Hz} ; 5$ a $10 \mathrm{~kg}: 8 \mathrm{~Hz}: 10$ a $20 \mathrm{~kg}: 6 \mathrm{~Hz} ; 20$ a $25 \mathrm{~kg}: 5$ $\mathrm{Hz}$; más de $25 \mathrm{~kg}$ : $4 \mathrm{~Hz}$ ); el tiempo inspiratorio se mantuvo en $33 \%$. Durante la ventilación de alta frecuencia se registraron los reglajes y los gases 30 min y $4,6,12,24$ y $48 \mathrm{~h}$ después de jniciar su aplicación. La meta inicial fue disminuir la $\mathrm{FiO}_{2}$ hasta 0,6 manteniendo Sat $\mathrm{O}_{2} \geq 90 \%$ para luego reducir la PMVA de 2 en $2 \mathrm{~cm} \mathrm{H}_{2} \mathrm{O}$. Si no se lograba aumentar la $\mathrm{Sat} \mathrm{O}_{2} \geq 90 \%$ se aumentó progtesivamente la PMVA de 2 en $2 \mathrm{~cm} \mathrm{H} \mathrm{H}_{2} \mathrm{O}$, controlando la hiperinsuflación pulmonar con radiogralia anteroposterior de tórax (diafragma hasta la novena vértebra dorsal o insuflación pulmonar no superior a nueve espacios intercostales) y vigilando que lós criterios hemodinámicos permanecieran estables. Se toletó $\mathrm{PaCO}_{2} \leq 60$ torr. Si era mayor se aumentó la $\Delta P$ en progresiones de $5 \mathrm{~cm} \mathrm{H}_{2} \mathrm{O}$; Juego se dismintyó la frecuencia respiratoria de $\mathrm{I}$ en I $\mathrm{Hz}$ (hasta un míniıno de 3 $\mathrm{Hz}$, aumentando posteriormente la $\Delta \mathrm{P}$ en $5 \mathrm{~cm} \mathrm{H} \mathrm{H}_{2} \mathrm{O}$. En caso de hipocapnia ( $\mathrm{PaCO}_{2} \leq 30$ torr). se redujo la $\Delta \mathrm{P}$ de 5 en $5 \mathrm{~cm}$ de $\mathrm{H}_{2} \mathrm{O}$. Si la ventilación era satisfactoria, Ia ftecuencia respirataria se mantuvo sin modificarla en todo el ciclo de alta frecueacia. Los pacientes fueron transferidos a apoyo convencional cuando se obtuvieron oxigenación y ventilación satisfactorias con $\mathrm{FiO}_{2} \leq 0,4$, PMVA $\leq 20 \mathrm{~cm}$ $\mathrm{H}_{2} \mathrm{O}$ y $\Delta \mathrm{P} \leq 30 \mathrm{~cm} \mathrm{H}_{2} \mathrm{O}$. El lubo endotraqueal fue aspirado desconectando el circuito y rentilando al paciente con bolsa tipo Jackson-Reese en márgenes de presion entre 20 y $30 \mathrm{~cm} \mathrm{H}_{2} \mathrm{O}$ medidas por manometria continua. Antes de reconectar el paciente a ventilación de alta frecuencia se le hacía inflación sostenida con $30 \mathrm{~cm} \mathrm{H}_{2} \mathrm{O}$ por $10 \mathrm{seg}$ para reclutar el volumen pulnonar perdido durante la desconexión.

Se consideró fracasado el ciclo de alta frecuencia si era inposible disminuir la $\mathrm{FiO}_{2}$ en $10 \%$ luego de $8 \mathrm{~h}$ de inciciado el procediriuento o mantener $\mathrm{PaCO}_{2}<80$ rorr con $\mathrm{pH}>7,2$.

Para comparar las variables en el tiempo se uso el analisis de varianza para medidas repetidas (ANOVA) y la prueba de comparaciones múltiples de Student-NewmanKeuls. Las comparaciones entre vivos y fallecidos se hicie. ron con la prueba de rangos de Wilcoxon.

\section{Resultados}

En la tabla se resurnen las características de los pacientes. El caso 3 era el único paciente en el cual se indicó el método debido a barotrauma y fue excluido del análisis final por sufrir una malformación cardiaca -con cirugía paliativaque impedía aplicar los criterios gasométricos. Los restantes pacientes (6 varones) presentaban un trastorno alveolar difuso, su peso era $9 \pm 8,2$ $\mathrm{kg}$ (márgenes 3,25 a $28,8 \mathrm{~kg}$ ), nueve tenían falla orgánica múltiple y en $10 \mathrm{el}$ índice de daño pulmonar agudo era grave $(>2,5)$. Antes de iniciar 
Tabla

Características de doce pacientes ventilados con alta frecuencia oscilatoria

\begin{tabular}{|c|c|c|c|c|c|c|c|c|c|}
\hline Caso & Edad & $\begin{array}{r}\text { Pess } \\
\text { (kg) }\end{array}$ & Diagnóstico & DDPA & $\mathrm{DA}-\mathrm{aO}_{2}{ }^{\mathrm{Al}^{\prime}}$ & $\begin{array}{l}\text { tes de VAI } \\
\text { IO } \\
\text { (mmBg) }\end{array}$ & $\begin{array}{l}\text { FO } \\
\text { Compromiso } \\
\text { de slstemgs }\end{array}$ & $\begin{array}{l}\text { Tiempo } \\
\text { (h) en } \\
\text { vaFo }\end{array}$ & Resultado \\
\hline 1 & $2,5 \mathrm{a}$ & 15,0 & $\begin{array}{l}\text { Asfixia por } \\
\text { inmersión }\end{array}$ & 3,3 & 441 & 33 & 2 & 60 & Vivo \\
\hline 2 & $6 \mathrm{ml}$ & 3,7 & $\begin{array}{l}\text { Hemonagia } \\
\text { pulmonar }\end{array}$ & 3,0 & 596 & 59 & 2 & 15 & Vivo \\
\hline 3 & $4 \mathrm{~m}$ & 5,2 & $\begin{array}{l}\text { Cardiopatia } \\
\text { cianotica } \\
\text { Barotrauma }\end{array}$ & $(-)$ & $(-)$ & $(-)$ & 2 & 24 & Vivo \\
\hline 4 & Ja & 10,8 & Histiocitosis & 2,6 & 469 & 23 & 4 & 60 & Vivo \\
\hline 5 & $5 \mathrm{~m}$ & 7,0 & Meningococcemia & 3,0 & 608 & 52 & 4 & 42 & Muerto \\
\hline 6 & $6 \mathrm{~m}$ & 4,1 & Neumonia & 3,0 & 547 & 42 & 4 & 56 & Vivo \\
\hline 7 & $3 \mathrm{ds}$ & 3.2 & $\begin{array}{l}\text { Neumonfa } \\
\text { Sepsis }\end{array}$ & 2,3 & 516 & 35 & 2 & 72 & Vivo \\
\hline 8 & $3 a$ & 16.3 & Leucenia & 3.0 & 589 & 46 & 2 & 132 & Vivo \\
\hline 9 & $8 \mathrm{a}$ & 28,8 & $\begin{array}{l}\text { S linfo } \\
\text { proliferativo }\end{array}$ & 3,3 & 569 & 57 & 5 & 28 & Muerto \\
\hline 10 & $22 \mathrm{ds}$ & 3,4 & $S$ aspirativo & 3.0 & 634 & 50 & 1 & 116 & Vivo \\
\hline 11 & $1 \mathrm{~m}$ & 3,0 & $\begin{array}{l}\text { CIV operada } \\
\text { Neumonfa }\end{array}$ & 3.0 & 562 & 40 & 3 & 60 & Muerto \\
\hline 12 & $2 \mathrm{~m}$ & 3,5 & Neumonia CMV & 3,0 & 566 & 25 & 1 & 264 & Vivo \\
\hline
\end{tabular}

IDPA : índice de daño pulmonar agudo

$\mathrm{DA} \mathrm{aO}_{2}:$ diferencia alvéolo arterial de oxígeno

IO : indice de oxigenación

VAFO : ventilación de alta frecuencia oscilatoria

$S \quad$ : sindrome

CIV : comunicación interventricular. CMV: citomegalovirus

ventilación de alta frecuencia el índice de oxigenación era $42 \pm 12,2$, la $\mathrm{PaO}_{2} / \mathrm{FiO}_{2} 59 \pm 18,6$ y la $\mathrm{AO}_{2}-\mathrm{aO}_{2} 553 \pm 61,5$.

La ventilación convencional que precedio a la de alta frecuencia había durado $40,6 \pm 46,8 \mathrm{~h}$ ( $6 \mathrm{~h}$ a 6 días). La duración de los ciclos de alta frectencia fue $82,3 \pm 69,3$ h ( 15 h a 11 días). No encontramos relación estadística entre la duración de estos períodos y la mortalidad. Los IO disminuyeron en forma sostenida y significativa entre el comienzo de la ventilación a alta frecuencia y las $4 \mathrm{~h}$ siguientes (ANOVA $p<0,0001$ ).

Tres pacientes fallecieron. Todos tenían falla orgánica múltiple y tres o más sistemas comprometidos; ninguno murió de causa respiratoria. En todos ellos, al iniciar la ventilación de alta frecuencia, los promedios IO y $\mathrm{AO}_{2}-\mathrm{aO}_{2}$ eran mayores $(49,9 \pm 8,4$ y $580 \pm 24,5)$ que en los sobrevivientes $(39,1 \pm 12,5$ y $54 \mathrm{l}, 7 \pm 70,5)$ (p > $0,25)$ si bien no se registraron diferencias significativas en los IO durante la aplicación del procedimiento (figura 2). Uno de ellos murí6 durante la VAFO, cuando estaba próximo a transferirse a apoyo convencional y dos estando en apoyo ventilatorio convencional. De los fallecidos en VMC, uno fue transferido desde VAFO de acuerdo con los criterios establecidos para ello, mientras en el otro se intentó VMC luego de $60 \mathrm{~h}$ de VAFO por respuesta no satisfactoria, aunque aún no cumplía criterios de fracaso. Los pacientes con buena respuesta a VAFO (10/11) tuvieron 10 menores que aquel con respuesta insuficiente (1/11) (figura 3), no siendo posible 


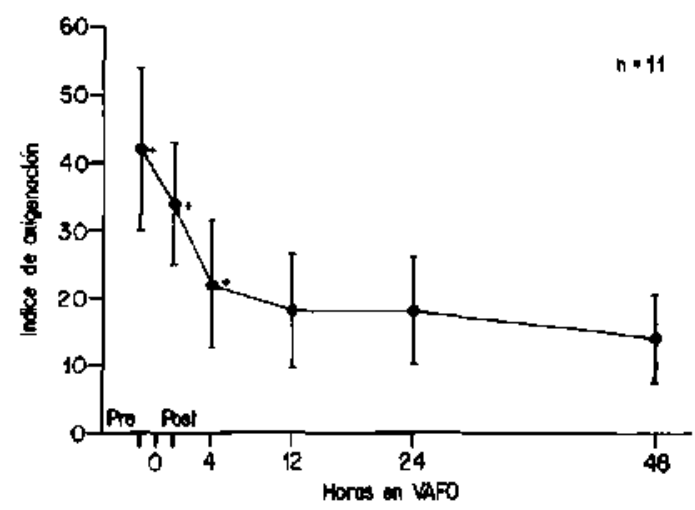

Figura 1: Indice de oxigenación (IO) antes (Pre) y durante las primeras $48 \mathrm{~h}$ de ventilación de alta frecuencia oscjlatoria (VAFO) en todos los pacientes. La disminución del 10 fue significativa entre el comienzo y las 4 h después de iniciar VAFO (ANOVA $p<0,000 t$ ).

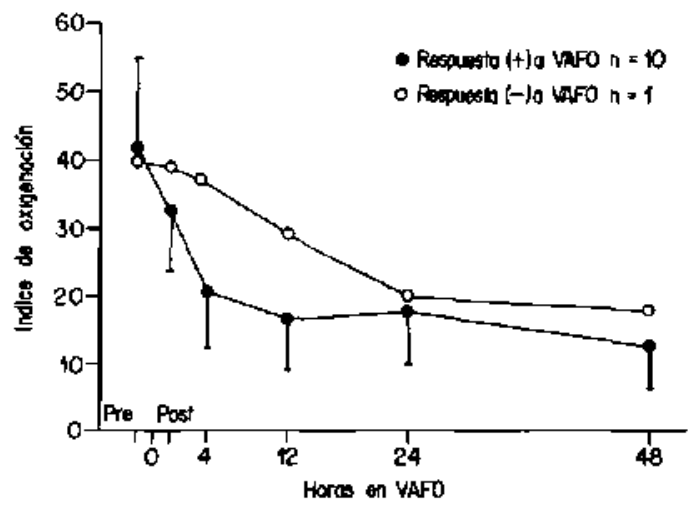

Figura 3: Evolución de los indices de oxigenación (10) durante las primeras $48 \mathrm{~h}$ de ventilación de alta frecuencia (VAFO) en pacientes con buena (círculos abiertos) y maja respuestas (círculos cerrados).

el análisis estadístico por el escaso tamaño de la muestra. En ninguno de los pacientes hubo problemas en el manejo de la ventilación, lográndose precozmente las $\mathrm{PaCO}_{2}$ deseadas durante el ciclo de VAFO. Todos los sobrevivientes $(8 / 11)$ pudieron convertirse a VMC y extubarse. No se observaron complicaciones atribuibles a VAFO.

\section{Comentario}

Los críterios gasométricos empleados en estos casos fueron más exigentes que en otras pu-

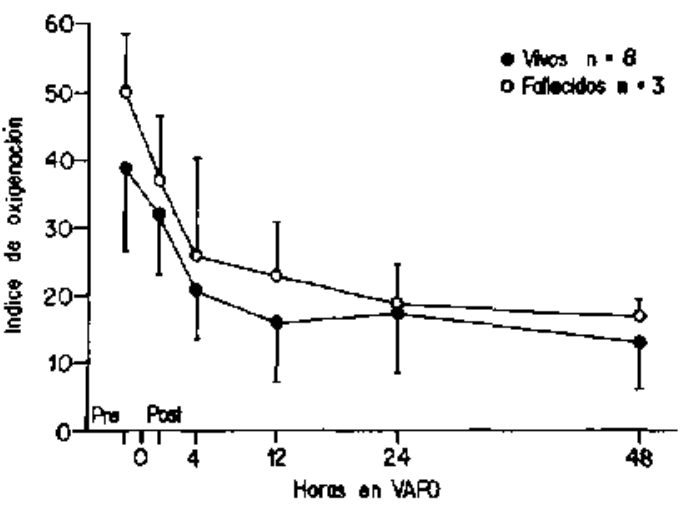

Figura 2: Indices de oxigenación (IO) durante las primeras $48 \mathrm{~h}$ de ventilacion de alta frecuencia oscilatoria (VAFO) en pacientes vivos (circulos abiertos) y fallecidos (círculos cerrados). No hubo diferencias significativas entre los IO antes (liempo "Pre") y después (tiempo "Post") de la conexion a VAFO.

blicaciones ${ }^{14.15}$. Todos ellos tenían un alto riesgo de mortalidad atribuible exclusivamente a su patología respiratoria ${ }^{18.20}$. Su mortalidad esperada superaba $70 \%^{18-21}$, a pesar de lo cual sobrevivieron y superaron la insuficiencia respiratoria $8 / 11$ pacientes $(72,7 \%)$ y en ninguno de los fallecidos la falla respiratoria tuvo relación inmediata con la muerte. La buena sobrevida coincide con otras publicaciones que analizan VAFO en IRA grave pediátrica ${ }^{12 \cdot 16}$.

La falta de diferencias significativas en la evolución de los IO entre sobrevivientes y fallecidos muestra la buena respuesta al procedimiento. Los cambios fundamentales del IO se producen durante las primeras $24 \mathrm{~h}$ de VAFO ${ }^{12,15}$. Las variaciones del IO tendrían un valor predictivo de mortalidad y, probablemente, de fracaso del método 15. 16; Arnold et al ${ }^{15}$ lograron establecer que aquellos pacientes con $\mathrm{IO} \geq 42$ luego de $24 \mathrm{~h}$ de VAFO presentaban alta mortalidad en forma estadísticamente significativa. En nuestra experiencia sólo en un paciente la respuesta a VAFO fue insatisfactoria.

Ninguno de los pacientes, vivos o fallecidos, presentó complicaciones atribuibles a la VAFO, lo que tambiên ha sido observado en otras publicaciones $^{12-15}$, siendo particularmente significativa la ausencia de complicaciones hemodinámicas, aspecto evaluado dirigidamente en el estudio de Gutiérrez et $a l^{22}$. Esto es un hecho relevante, pues teóricamente es una de Jas complicaciones más esperadas de la VAFO? 
El control de la ventilación es un factor limitante de la indicación de VAFO, pero cuando los pacientes son seleccionados adecuadamente no hay problemas en el manejo de la $\mathrm{PaCO}_{2}$, determinándose en forma relativamente precoz las FR y $\Delta \mathrm{P}$ óptimas del oscilador. La hiperinsuflación pulmonar tampoco fue un problema significativo. Aunque no fue posible evaluar el resultado de la VAFO en el tratamiento del barotrauma, el único paciente con este diagnóstico tuvo una evolución favorable.

Estos resultados, en coincidencia con otras publicaciones, permiten concluir que la VAFO es una alternativa eficaz, segura y poco invasiva para el tratamiento del SDRA pediátrico, constituyendo una herramienta nueva, asequible, menos compleja y más económica en comparación a técnicas como la oxigenación extracorpórea a través de membrana o ventilación líquida, 23,24 , las cuales aún no están disponibles en nuestro medio. La VAFO debe considerarse como un complemento de la VMC y estaría indicada en el pequeño grupo de pacientes en que es preciso emplear reglajes considerados pelígrosos. Además de esta indicación de "rescate", queda por definir su rol en la prevención del daño pulmonar secundario a ventilación mecánica cuando es indicada precozmente en el curso del SDRA.

Aunque los resultados de este y otros trabajos son promisorios, es necesario desarrollar nuevos estudios controlados y acumular más experiencia para definir adecuadamente el rol de la VAFO en el tratamiento del SDRA pediátrica y en la prevención del daño pulmonar secundario a VM, así como su empleo asociado a otras terapias de introducción reciente, como el óxido nítrico y surfactante pulmonar ex 6 geno $0^{5 \cdot 6.25-27}$.

\section{Referencias}

1. Chang HK: Mechanisıns of gas transport during ventilation by high frequency oscillation. 1 Appl Physiol 1984: 56: 553-563.

2. Wetzel $R C$, Gisia FR: High frequency ventilation. Pediatr Clin North Am 1987: 34: 15-38.

3. Hernandez LA, Peevy KJ, Moise RA, Parker JC: Chest wall restriction limits high airway pressure-induced lung injury in young rabbits. J Appl Physiol 1989; 66: 2364-2368

4. Parker JC. Herntindez LA. Peevy KJ: Mechanisms of ventilator-induced lung injury. Crit Care Med 1993; 21: 131-143.
5. Ring JC. Stidham GL: Novel therapies for acute respiratory failure. Pediatr Clin North Am I994; 41: 1325-1363.

6. Pauison TE. Spear RM, Peterson BM: New concepts in the treatment of children with acute respiratory distress syndrome. J Pediarr 1995: 127: 163-175.

7. Kodríguez J, Ronco R, Carfillo A: Ventilación de alta frecuencia en pediatria. Rev Chil Pediatr 1997: 68: 274-279.

8. The HIFf Study Group: High-frequency oscillalory ventilation compared with conventional mechanical ventilation in the treatment of respiralory fajlute in preterm infants. N Engl J Med 1989; 320: 88-93.

9. Clark RH, Gerstmann DR. Nall DM Jr, et al: Prospective randomized comparison of high frequency oscillatory and conventional ventilation in respiratory distress syndrome. Pediatrics 1992; 89: 5.12.

10. HIFO Sudy Group: Randomized study of highfrequency oscillalory ventilation in infants with severe respiratory distress syndrome. J Pediats 1993; I22: 609.619.

I1. Clark RH: High-frequency ventilation. J Pediatr 1994; 124: $661-670$.

12. Arnold JH. Truog RD, Thompson JE. et al: Highfrequency oscillatory ventilation in pediatric respiralory failure. Cril Care Med 1993:21: 272-278.

13. Cappon J, Anas N: High-frequency oscillatory ventilation in ECMO-elegible children with severe respiratory failure Am Rev Respir Dis 1993; 147 (Suppl): A887.

14. Rosenberg RB. Broner CW. Peiers KJ, et al: Highfrequency ventilation for acute pediatric respiratory failure. Chest 1993; 104: 1216-1221.

15. Arnold $\mathrm{JH}$, Honson JH. Toro-Figwero LO, et ali Prospective, randomized comparison of high-frequency oscillatory ventilation and conventional unechanical venlilation in pediatric respiratory failure. Crit Care Med 1994: 22: 1530-1539.

16. Sarnaik AP. Meerr KL, Pappas MD, et al: Predicting outcome in children with severe acute respiralory failure treated with high-frequency ventilation. Crit Care Med 1996; 24: 1396-1402.

17. Konco $R$, Rodriguez J. Castillo $A$, et al: Ventilación de alta frecuencia oscilatoria en SDRA pediátrico: Informe preliminar. Libro de resimenes Tercer Congreso Latinoamericano de Emergencias y Cuidados Inıensivos Pediátricos. Viña de] Mar, 1997:74.

18. Murray JF, Matthay MA, Luce JM: An expanded definition of the adult respiratory distress syndrome. Aлn Rev Respir Dis 1988; 138: 720-723.

19. Wilkinson JD, Pollack MM, Glass NL, ef at: Mortalily associated with multiple organ system failure and sepsis in a pediatric intensive care unit. J Pediatr 1987; [1]: 324

20. Wifkinson JD. Pollack MM. Ruttimunn UE, el at: Outcome of pediatric patients with multiple organic system failure, Crit Care Med 1986; 14:27i-274.

21. Davis SL, Furman DP. Costarinn AT: ARDS in children: associated disease, clinical course, and predictors of death. J Pediatr 1993: 123: 35-45.

22. Gutiérrez $J N$, Levin $D L$, Toro-Figuerra LO: Hemodyramic effects of high frequency oscillatory ventilation in severe pediatric respiratory failute. Intensive Care Med 1995:21: 505-510. 
23. Peurson GA, Grant J, Field D. et al: Extracorporeal life support in pediatrics. Arch Dis Child 1993; 68: 94 96.

24. Leach CI, Futrmen BP, horis FC. ef al: Perflurocarbon-associated gas exchange (pustial liquid ventila(ion) in respiratory disıress syndrome: A prospective, randomized, controlled sudy. Crit Care Med J993; 21: 1270-1278.

25. Fulkersan $W$, Macintyre $N$, Stamler $J_{\text {, et }}$ al: Pathogenesis and reatment of the adult respiratory distress syndrome. Arch Intern Med 1996; 156: 2938.

26. Demirakca S. Dötsch J. Knothe $C$, et at: Inhaled nitric oxide in neonatal and pediatric acute respiratory distress syndrome: Dose response, prolonged inhalation, and weaning. Crit Care Med 1996: 24: 1913-1919.

27. Gregory TJ. Steinberg KP, Spragg $R$, et al: Bovine surfactant therapy for patients with acute respiratory distress syndrome. Am J Respir Crit Care Med 1997; 155: $1309-1315$

\section{AVISO A LOS AUTORES}

Por acuerdo del Comité Editorial, la Revista Chilena de Pediatría devolverá sin tramitar todos los trabajos que no den estricto cumplimiento al Reglamento de Publicaciones y a las Instrucciones de los Autores que se editan en cada número de la Revista. 